\title{
VALUES IN DIFFERENTIAL GAMES ${ }^{1}$
}

\author{
BY JOHN M. DANSKIN
}

Communicated by R. C. Buck, March 5, 1973

\begin{abstract}
This is an announcement of results to be proved in detail in a later report in book form.

The author introduces a new value, the $\Omega$-value, into differential games, and proves that six well-known values for games of perfect information now in the literature are special cases of it. He proves that two (in the present slightly altered formulation four) values introduced in 1972 by Elliott and Kalton using relaxed controls are equal to the mixed-strategy value introduced by Fleming, in 1964. Thus, of the twelve values just mentioned, there are only two which are essentially distinct: the new $\Omega$-value, and Fleming's 1964 value.

Hamilton-Jacobi equations for the $\Omega$-value and for the Fleming mixed-strategy value are announced as well.
\end{abstract}

1. Introduction. We characterize a differential game of unit duration of purely terminal type by the following data:

(i) $U$ and $V$, compact topological spaces;

(ii) $\mathrm{f}(x, t, u, v)$, a uniformly bounded vector-valued function continuous on $R^{p} \times[0,1] \times U \times V$ and uniformly Lipschitzian in $x$ and $t$;

(iii) $\varphi(x)$, a uniformly bounded and uniformly Lipschitzian function defined throughout $R^{p}$.

There are several methods already in the literature for attaching a value to this differential game. We begin by proposing a new one.

2. The $\Omega$-value. Let $0 \leqq \sigma \leqq 1$. We conceive of $\sigma$ as the proportionate reaction time for the maximizing player. Put $t_{n}=n / N$, and $t_{n}^{\sigma}=t_{n}+$ $(1-\sigma) / N, n=0, \cdots, N-1$. At time $t_{0}$ the maximizer chooses $u_{0} \in U$. A predecessor $v_{-1}$ is supposed given a priori at that time. Then, at time $t_{0}^{\sigma}$, the minimizer chooses $v_{0} \in V$, and so on. Suppose that at time $t_{n}$ the position vector, having started at time $t_{0}$ at a starting point $x_{0}$, reaches $x_{n}$. The maximizer now chooses $u_{n}$, and the position vector, starting at $\mathfrak{X}\left(t_{n}\right)=x_{n}$, follows the differential equation

$$
\dot{\mathfrak{X}}(t)=\mathfrak{f}\left(\mathfrak{X}(t), t, u_{n}, v_{n-1}\right)
$$

on $\left[t_{n}, t_{n}^{\sigma}\right)$, reaching $x_{n}^{\sigma}=\mathfrak{x}\left(t_{n}^{\sigma}\right)$ at time $t_{n}^{\sigma}$. The minimizer now chooses

AMS (MOS) subject classifications (1970). Primary 90D25.

${ }^{1}$ Research supported by the U.S. Air Force Office of Scientific Research under Grant No. 1924-10, and by the Mathematics Research Center of the University of Wisconsin under U.S. Army contract DA-31-124-ARO-D-462. 
$v_{n}$, and the position vector, starting at $x_{n}^{\sigma}$, follows the differential equation

$$
\dot{\mathfrak{x}}(t)=\mathfrak{f}\left(\mathfrak{x}(t), t, u_{n}, v_{n}\right)
$$

on $\left[t_{n}^{\sigma}, t_{n+1}\right)$, reaching $x_{n+1}=\mathfrak{x}\left(t_{n+1}\right)$ at time $t_{n+1}$. This process continues until time $t_{N}=1$ is reached; the position vector is now at $x(1)$. The payoff is then $\varphi(\mathfrak{x}(1))$. Put

$$
\Omega_{N}\left(\sigma, v_{-1}, x_{0}\right)=\underset{u_{0}}{\operatorname{Max}} \operatorname{Min}_{v_{0}} \cdots \underset{u_{N-1}}{\operatorname{Max}} \operatorname{Min} \varphi(x(1)) .
$$

This is the value of the $N$-stage $\Omega$-game.

In a book [1] currently being prepared for publication we have proved the following.

ConvergenCe THEOREM. $\Omega_{N}\left(\sigma, v_{-1}, x_{0}\right)$ converges uniformly in all its variables as $N \rightarrow \infty$ to a continuous limit $\Omega\left(\sigma, x_{0}\right)$.

The proof of this theorem is very long and involves delicate measuretheoretical and probabilistic arguments.

The simplest linear $\Omega$-problem. Consider the trivial case in which the control function $\overline{\mathfrak{f}}(u, v)$ does not involve $x$ and $t$, the starting point is $x_{0}=0$, and the payoff is $\varphi(x)=\mathfrak{a} \cdot x, \mathfrak{a}$ being a constant vector in $R^{p}$. We denote the value of this differential game by $\bar{w}(\sigma, \mathfrak{a})$. Evidently

(4) $\quad \bar{w}(0, \mathfrak{a})=\operatorname{Min}_{v} \operatorname{Max}_{u} \mathfrak{a} \cdot \bar{f}(u, v), \quad \bar{w}(1, \mathfrak{a})=\operatorname{Max}_{u} \operatorname{Min}_{v} \mathfrak{a} \cdot \bar{f}(u, v)$.

If $0<\sigma<1$ the value satisfies $\bar{w}(0, \mathfrak{a}) \geqq \bar{w}(\sigma, \mathfrak{a}) \geqq \bar{w}(1, \mathfrak{a})$, and the problem of finding the solution is in general much more complicated than it is for $\sigma=0$ or 1 .

The Hamilton-Jacobi equation for the $\Omega$-game. Let $t \in[0,1)$. Denote by $\Omega(t, \sigma, x)$ the value of the $\Omega$-game starting at time $t$ instead of at time 0 ; in defining $\Omega_{N}$ one divides $[t, 1]$, instead of $[0,1]$, into $N$ segments. By a 1919 theorem of Rademacher ([7]; a proof will be found in [6]), $\Omega(t, \sigma, x)$, since it is Lipschitzian in $t$ and $x$, has a total derivative almost everywhere in $[0,1] \times R^{p}$. Let $(\bar{t}, \bar{x})$ be a point of total differentiability. Put $\mathfrak{a}=\nabla_{x} \Omega(\bar{t}, \sigma, \bar{x})$, and $\overline{\mathfrak{f}}(u, v)=\mathfrak{f}(\bar{x}, \bar{t}, u, v)$. Then

$$
\Omega_{t}(\bar{t}, \sigma, \bar{x})=-\bar{w}(\sigma, \mathfrak{a}),
$$

the subscript $t$ denoting the partial derivative and $\bar{w}$ being defined as above. This is the Hamilton-Jacobi equation for the $\Omega$-problem. It has not been proved before even for the case of the Fleming upper value $(\sigma=0)$ or the Fleming lower value $(\sigma=1)$, at least not for general Lipschitzian terminal $v$.

3. Other values. We enumerate eleven important values already in the literature. 
(i) The Fleming upper value. This is the same as $\Omega\left(0, x_{0}\right)$, as follows from the definition. It was defined in [4]. We denote the $N$-stage value by $\Phi \lambda_{N}^{+}\left(x_{0}\right)$. Except for the very special case considered in [4], Fleming has not published an explicit proof of convergence. Elliott and Kalton knew how to prove it by the time they wrote [3], where however they attribute it to Fleming. They restrict $N$ to powers of 2 . Since

$$
\Phi \lambda_{N}^{+}\left(x_{0}\right)=\operatorname{Min}_{v_{-1}} \Omega_{N}\left(0, v_{-1}, x_{0}\right),
$$

this convergence in fact holds as $N \rightarrow \infty$ through all values.

(ii) The Fleming lower value. This is the same as $\Omega\left(1, x_{0}\right)$, and is denoted by $\Phi \lambda-\left(x_{0}\right)$.

The objective of the introduction of the $\Omega$-value in the first place was to bridge the gap between Fleming's upper and lower values for the perfect information case.

(iii) The Friedman upper value. Put $t_{n}=n / N, n=0, \cdots, N-1$. Denote by $\mathfrak{U}_{n}$ the set of all Borel-measurable functions $\mathfrak{u}_{n}$ from $\left[t_{n}, t_{n+1}\right)$ to $U$, and define $\mathfrak{B}_{n}=\left\{\mathfrak{v}_{n}\right\}$ similarly. Suppose the position vector starts at time $t_{0}$ at $x_{0}$, and that it has reached $x_{n}$ by the time $t_{n}, n \leqq N-1$. The position vector, starting with $\mathfrak{x}\left(t_{n}\right)=x_{n}$, now follows the differential equation

$$
\mathfrak{X}(t)=\mathfrak{f}\left(\mathfrak{x}(t), t, \mathfrak{u}_{n}(t), \mathfrak{v}_{n}(t)\right)
$$

on $\left[t_{n}, t_{n+1}\right)$, reaching a point $x_{n+1}=\mathfrak{x}\left(t_{n+1}\right)$. At time 1 the position vector reaches a point $\mathfrak{x}(1)$. Put

$$
F_{N}^{+}\left(x_{0}\right)=\operatorname{Inf}_{\mathfrak{v}_{0}} \operatorname{Sup}_{\mathfrak{u}_{0}} \cdots \operatorname{Inf}_{\mathfrak{v}_{N-1}} \operatorname{Sup}_{\mathfrak{u}_{N-1}} \varphi(\mathfrak{x}(1)) .
$$

This is the Friedman upper value. Friedman gives a more complicated but equivalent definition on p. 11 of [6]. He proves in [6] that $F_{N}^{+}\left(x_{0}\right)$ converges uniformly to a limit $F^{+}\left(x_{0}\right)$ as $N$ tends to $\infty$ through all values. We give another proof of this in [1].

(iv) The Friedman lower value. The same, except that it is

$$
\text { Sup Inf } \cdots \text { Sup Inf. }
$$

(v) The Varaiya-Roxin upper value. Denote by $\mathfrak{U}=\{\mathfrak{u}\}$ the set of all Borel-measurable functions from $[0,1]$ to $U$; denote $\mathfrak{B}=\{\mathfrak{p}\}$ similarly. Let

$$
\alpha: \mathfrak{B} \rightarrow \mathfrak{U}
$$

be any function satisfying the "no-foreknowledge" condition that

$$
\mathfrak{v}(\tau)=\mathfrak{v}^{\prime}(\tau) \text { a.e. on }[0, t] \Rightarrow \alpha \mathfrak{v}(\tau)=\alpha \mathfrak{v}^{\prime}(\tau) \text { a.e. on }[0, t] .
$$

Let $x_{0}$ be a starting point and let $\mathfrak{u}, \mathfrak{v}$ be arbitrary in $\mathfrak{U}, \mathfrak{B}$. Let $\mathfrak{x}(1)$ be the point reached by following the differential equation

$$
\dot{\mathfrak{X}}(t)=\mathfrak{f}(\mathfrak{x}(t), t, \mathfrak{u}(t), \mathfrak{v}(t))
$$


to time 1 , starting at $\mathfrak{x}(0)=x_{0}$. Put $\Theta\left(x_{0}, \mathfrak{u}, \mathfrak{v}\right)=\varphi(\mathfrak{x}(1))$. The VaraiyaRoxin upper value of the differential game is

$$
V R^{+}\left(x_{0}\right)=\operatorname{Sup}_{\alpha} \operatorname{Inf}_{\mathfrak{v}} \Theta\left(x_{0}, \alpha \mathfrak{v}, \mathfrak{v}\right) .
$$

This was defined first in 1968 in [9] and extended in 1968 in [8]. It has the advantage of not needing convergence proofs.

(vi) The Varaiya-Roxin lower value $V R^{-}\left(x_{0}\right)$ is defined similarly.

(vii) The Fleming mixed-strategy value. This was defined by Fleming in 1964, in [5]. We shall define his $N$-stage value here by backward induction; this is different from his definition but is equivalent. Let $t_{n}=n / N, n=0, \cdots, N$. Put $\Phi \lambda_{N}\left(x_{N}, t_{N}\right)=\varphi\left(x_{N}\right)$. Now suppose $n \leqq N-1$ and that $\Phi \lambda_{N}\left(x_{n+1}, t_{n+1}\right)$ has already been defined throughout $R^{p}$. Denote by $X_{n+1}\left(x_{n}, u_{n}, v_{n}\right)$ the point reached by starting at $\mathfrak{x}\left(t_{n}\right)=x_{n}$ at time $t_{n}$ and following the differential equation

$$
\dot{\mathfrak{X}}(t)=\mathfrak{f}\left(\mathfrak{X}(t), t, u_{n}, v_{n}\right)
$$

to time $t_{n+1}$; thus $X_{n+1}\left(x_{n}, u_{n}, v_{n}\right)=\mathfrak{x}\left(t_{n+1}\right)$. Put

$$
\mathfrak{f}_{n}\left(x_{n}, u_{n}, v_{n}\right)=\Phi \lambda_{N}\left(X_{n+1}\left(x_{n}, u_{n}, v_{n}\right), t_{n+1}\right) .
$$

For each $x_{n}$ this defines a continuous game over $U \times V$. We denote the value of this game by $\Phi \lambda_{N}\left(x_{n}, t_{n}\right)$. When we reach time $t_{0}=0$ we put $\Phi \lambda_{N}\left(x_{0}\right)=\Phi \lambda_{N}\left(x_{0}, t_{0}\right)$. This is the Fleming $N$-stage value of the differential game with mixed strategies.

Fleming proved in [5] that $\Phi \lambda_{N}\left(x_{0}\right)$ converges as $N \rightarrow \infty$ through all values to a limit $\Phi \lambda\left(x_{0}\right)$, and that the convergence is uniform on bounded sets. He used deep techniques from the theory of parabolic differential equations and of probability.

We give in [1] a new proof of Fleming's convergence theorem, not involving the parabolic equation. We also give a proof that the HamiltonJacobi equation for this problem holds almost everywhere in $[0,1] \times R^{p}$. This result, for arbitrary uniformly Lipschitzian $\varphi$, is new.

(viii) The Elliott-Kalton upper value. This is the $V_{12}^{+}$defined in [3] just preceding Theorem 8.4. This time, on $\left[t_{n}, t_{n+1}\right)$ the position vector follows the differential equation

$$
\dot{\mathfrak{X}}(t)=\iint \mathfrak{f}(\mathfrak{X}(t), t, u, v) d \mathfrak{P}_{t}^{n}(u) d \mathfrak{Q}_{t}^{n}(v),
$$

$\mathfrak{P}^{n}$ and $\mathfrak{Q}^{n}$ being "relaxed controls" on $\left[t_{n}, t_{n+1}\right)$, i.e. functions from $\left[t_{n}, t_{n+1}\right)$ into the spaces of probability measures on $U$ and $V$ respectively, satisfying the condition of "scalar measurability" relative to $t$. Relaxed controls had been introduced into control theory by Warga; see his 
book [10]. We put

$$
E K_{N}^{+}\left(x_{0}\right)=\operatorname{Inf}_{\mathbb{Q}^{0}} \operatorname{Sup}_{\mathfrak{P}^{0}} \cdots \operatorname{Inf}_{\mathfrak{Q}^{N-1}} \operatorname{Sup}_{\mathfrak{P}^{N-1}} \varphi(\mathfrak{X}(1)) .
$$

This is the Elliott-Kalton $N$-stage value of the differential game. For technical reasons this definition is slightly different from that given in [3]; but it comes down to the same thing as $N \rightarrow \infty$. Elliott and Kalton prove in [3] that $E K_{N}^{+}\left(x_{0}\right)$ converges as $N \rightarrow \infty$ through powers of 2 to a limit $E K^{+}\left(x_{0}\right)$. We prove in [1] that this convergence holds as $N \rightarrow \infty$ through all values.

(ix) The Elliott-Kalton lower value is defined similarly.

(x) The Varaiya-Roxin-Elliott-Kalton upper value. Denote by $\{\mathfrak{P}\}$ and $\{\mathfrak{Q}\}$ respectively the spaces of relaxed controls over $[0,1]$ for the maximizing and minimizing players. Denote by $\mathfrak{A}:\{\mathfrak{Q}\} \rightarrow\{\mathfrak{P}\}$ a function satisfying the "no-foreknowledge" condition analogous to that given for $\alpha$ at (8). With a slight abuse of notation, we denote by $\Theta\left(x_{0}, \mathfrak{P}, \mathfrak{Q}\right)$ the value $\varphi(\mathfrak{x}(1))$ attained by a position vector starting at time 0 with $\mathfrak{x}(0)=0$ and following the differential equation

$$
\dot{\mathfrak{x}}(t)=\iint \mathfrak{f}(\mathfrak{x}(t), t, u, v) d \mathfrak{P}_{t}(u) d \mathfrak{Q}_{t}(v)
$$

to time 1 . The quantity

$$
V R E K^{+}\left(x_{0}\right)=\operatorname{Sup}_{\mathfrak{A}} \operatorname{Inf}_{\mathfrak{Q}} \Theta\left(x_{0}, \mathfrak{A} \mathfrak{Q}, \mathfrak{Q}\right)
$$

is the Varaiya-Roxin-Elliott-Kalton upper value of the differential game. It was defined, somewhat tacitly, in Theorem 11.2 in [3].

(xi) The Varaiya-Roxin-Elliott-Kalton lower value is defined similarly.

4. Equality theorems. Elliot and Kalton proved in [3] that

$$
\Phi \lambda-\left(x_{0}\right) \leqq F^{-}\left(x_{0}\right) \leqq F^{+}\left(x_{0}\right) \leqq \Phi \lambda^{+}\left(x_{0}\right) .
$$

The inside inequality is of course trivial. They also proved that

$$
F^{-}\left(x_{0}\right) \leqq V R^{-}\left(x_{0}\right), \quad V R^{+}\left(x_{0}\right) \leqq F^{+}\left(x_{0}\right) .
$$

They did not suggest any relation between $V R^{-}\left(x_{0}\right)$ and $V R^{+}\left(x_{0}\right)$. Finally they proved that

$$
\operatorname{VREK}^{+}\left(x_{0}\right)=\operatorname{VREK}^{-}\left(x_{0}\right)=E K^{+}\left(x_{0}\right)=E K^{-}\left(x_{0}\right) .
$$

We may now write simply $\operatorname{VREK}\left(x_{0}\right), E K\left(x_{0}\right)$.

In [1] we have proved the following. 
THEOREM. For the perfect information case,

$$
\Phi \lambda^{+}\left(x_{0}\right)=F^{+}\left(x_{0}\right)=V R^{+}\left(x_{0}\right)=\Omega(0,1),
$$

and similarly for the lower values. In particular, $V R^{+}\left(x_{0}\right) \geqq V R^{-}\left(x_{0}\right)$.

Elliott and Kalton have obtained the first two equalities independently, using parabolic partial differential equation theory; their paper will appear as [11].

THEOREM. For the "probabilistic" case

$$
\operatorname{VREK}\left(x_{0}\right)=E K\left(x_{0}\right)=\Phi \lambda\left(x_{0}\right) .
$$

The first equality in (22), as we noted above, was proved by Elliott and Kalton.

Thus, there are only two values which are essentially distinct, the 1964 Fleming value $\Phi \lambda\left(x_{0}\right)$ for the mixed strategy case, and the new value $\Omega\left(\sigma, x_{0}\right)$.

5. The Isaacs condition. In the sense usually understood, the Isaacs condition requires that

$$
\operatorname{Max}_{u} \underset{v}{\operatorname{Min}} \mathfrak{a} \cdot \mathfrak{f}(x, t, u, v)=\underset{v}{\operatorname{Min}} \operatorname{Max}_{u} \mathfrak{a} \cdot \mathfrak{f}(x, t, u, v)
$$

for all vectors $\mathfrak{a} \in R^{p}$ and all pairs $x, t$. It is sometimes given another meaning by Elliott and Kalton in [3]; we adhere to (23).

Fleming proved in [4] that if $\mathfrak{f}$ is separable, i.e. if $\mathfrak{f}(x, t, u, v)=$ $\mathfrak{f}_{1}(x, t, u)+\mathfrak{f}_{2}(x, t, v)$, then $\Phi \lambda^{+}\left(x_{0}\right)=\Phi \lambda-\left(x_{0}\right)$. Friedman proved in [6] under the same hypothesis that $F^{+}\left(x_{0}\right)=F^{-}\left(x_{0}\right)$. Elliott and Kalton proved in [3] that if the Isaacs condition (23) is satisfied then $F^{+}\left(x_{0}\right)=$ $F^{-}\left(x_{0}\right)$. Under the further hypothesis that the terminal function $\varphi$ has Lipschitzian second partial derivatives, they proved that $\Phi \lambda^{+}\left(x_{0}\right)=\Phi \lambda-\left(x_{0}\right)$. Other than the Isaacs condition, the following theorem assumes only the hypotheses of $\S 1$.

THEOREM. If the Isaacs condition (22) is satisfied, all the values we have mentioned are equal: for all $\sigma \in[0,1]$

$$
\begin{aligned}
\Omega\left(\sigma, x_{0}\right) & =\Phi \lambda^{+}\left(x_{0}\right)=\Phi \lambda^{-}\left(x_{0}\right)=F^{+}\left(x_{0}\right)=F^{-}\left(x_{0}\right)=V R^{+}\left(x_{0}\right) \\
& =V R^{-}\left(x_{0}\right)=\Phi \lambda\left(x_{0}\right)=E K^{+}\left(x_{0}\right)=E K^{-}\left(x_{0}\right) \\
& =V R E K^{+}\left(x_{0}\right)=V R E K^{-}\left(x_{0}\right) .
\end{aligned}
$$

There is a partial converse. Recall the definition of $\Omega(t, \sigma, x)$ at the beginning of the discussion of the Hamilton-Jacobi equation in $\$ 2$. 
THEOREM. If $\Omega(t, 0, x)=\Omega(t, 1, x)$ for all pairs $(t, x)$, then (22) is satisfied almost everywhere in $[0,1] \times R^{p}$, with $\mathfrak{a}=\nabla \Omega(t, 0, x)=\nabla \Omega(t, 1, x)$.

This follows trivially from the Hamilton-Jacobi equation (6).

6. Generalizations. All the above existence and equality results generalize to the case when $\mathfrak{f}$ is bounded, continuous in all its variables, and satisfies the weakened Lipschitz condition

$$
\left|\mathfrak{f}(x, t, u, v)-\mathfrak{f}\left(x^{\prime}, t, u, v\right)\right| \leqq k(t)\left|x-x^{\prime}\right|,
$$

where $\int_{0}^{1} k(t) d t<\infty$, and the terminal function $\varphi$ is replaced by a continuous functional $\Phi$ on the space $\mathfrak{X}$ of trajectory functions $\mathfrak{x}$. These are the most general conditions envisaged by Friedman in [6] or by Elliott and Kalton in [3]. There is of course no longer any Hamilton-Jacobi equation, and the last theorem in $\$ 5$ does not apply.

\section{REFERENCES}

1. John Danskin, Value in differential games (to appear).

2. Robert Elliott and Nigel Kalton, Values in differential games, Bull. Amer. Math. Soc. 78 (1972), 427-431. MR 45 \#4840.

3. - The existence of value in differential games, Mem. Amer. Math. Soc. No. 126 (1972).

4. Wendell Fleming, The convergence problem for differential games, J. Math. Anal. Appl. 3 (1961), 102-116. MR 25 \#5806.

5. - The convergence problem for differential games. II. Advances in game theory, Ann. of Math. Studies, no. 52, Princeton Univ. Press, Princeton, N.J., 1964, pp. 195210. MR 29 \#6947.

6. Avner Friedman, Differential games, Wiley, New York, 1971.

7. Hans Rademacher, Über partielle und totale Differenzierbarkeit von Funktionen mehrer Variabeln und über die Transformationen der Doppelintegrale. I, II, Math. Ann. 79 (1919), 340-359; ibid 81 (1920), 52-63.

8. Emilio Roxin, Axiomatic approach to differential games, J. Optimization Theory Appl. 3 (1969), 153-163. MR 39 \#5167.

9. P. P. Varaiya and J. Lin, Existence of saddle points in differential games, University of California at Berkeley, Electronics Research Laboratory, Memorandum No. ERL-M241, 1969.

10. Jack Warga, Optimal control of differential and functional equations, Academic Press, New York, 1972.

11. R. J. Elliott and N. J. Kalton, Upper values of differential games, J. Differential Equations 14, No. 1, (1973), 89-100.

Mathematics Research Center, University of Wisconsin, Madison, Wisconsin 53706

Current address: Electronics Research Laboratory, University of California, Berkeley, California 94720 